\title{
A method for cognitive 3D geological voxel modelling of AEM data
}

\author{
Flemming Jørgensen • Rasmus Rønde Møller • \\ Lars Nebel • Niels-Peter Jensen • Anders Vest Christiansen • \\ Peter B. E. Sandersen
}

Received: 20 November 2012/ Accepted: 14 May 2013

(c) Springer-Verlag Berlin Heidelberg 2013

\begin{abstract}
Airborne electromagnetic (AEM) data have proven successful for the purpose of near-surface geological mapping and are increasingly being collected worldwide. However, conversion of data from measured resistivity to lithology is not a straightforward task. Therefore, it is still challenging to make full use of these data. Many limitations must be considered before a successful geological interpretation can be performed and a reasonable 3D geological model constructed. In this paper, we propose a method for 3D geological modelling of AEM data in which the limitations are jointly considered together with a cognitive and knowledge-driven data interpretation. The modelling is performed iteratively by using voxel modelling techniques with tools developed for this exact purpose. Based on 3D resistivity grids, the tools allow the geologist to select voxel groups that define any desirable volumetric shape in the 3D model. Recent developments in octree modelling ensure exact modelling with a limited number of voxels.
\end{abstract}

Keywords Three-dimensional geological model . Airborne electromagnetic data · Voxel modelling . Octree $\cdot$ Groundwater

F. Jørgensen ( $\varangle)$ · R. R. Møller · P. B. E. Sandersen Geological Survey of Denmark and Greenland, GEUS, Lyseng Alle 1, 8270 Højbjerg, Denmark

e-mail: flj@geus.dk

L. Nebel · N.-P. Jensen

I-GIS A/S, Voldbjergvej 14A, 1, 8240 Risskov, Denmark

A. V. Christiansen

Department of Geoscience, Aarhus University,

C. F. Møllers Alle 4, 8000 Aarhus C, Denmark

\section{Introduction}

A 3D geological model, also referred to as a geoscientific information system (GSIS) (Turner 2006), is basically a spatial extension of a 2D geological map. Two-dimensional geological maps have been drawn for centuries, but as computer technology improves and dedicated, advanced 3D software is being developed, digital 3D geological modelling has become a widespread tool within geosciences and administration over the past 20 years.

The geology of glaciated areas is often complex, and detailed models are needed, for example, where the goal of a 3D model is to predict groundwater pathways to well fields (Troldborg et al. 2007, 2008). Due to the complexity of these areas, existing borehole data seldom provide an adequate data basis for the modelling, even if the geologist is highly experienced and makes adept use of background knowledge. This is one of the reasons for the growing use of geophysical methods with groundwater studies; especially, airborne electromagnetic methods (AEMs) supplement geological knowledge in important ways because they offer dense spatial information while at the same time provide information about lithological properties through measured electrical resistivity (Sandersen et al. 2009; Jørgensen et al. 2012; Oldenborger et al. 2013).

Three-dimensional geological models can be general models or purpose-specific models. The former are built for no other purposes than to describe the geometry of the geological layers and structures as well as their geological properties. By means of $3 \mathrm{D}$ visualisation and iterative model construction, such general models can be helpful in the spatial understanding and reconstruction of geology. Therefore, they are vital tools within certain research fields. Furthermore, they can serve as digital archives for current geological knowledge, even on a national scale (Stafleu 
et al. 2011). The purpose-specific models are constructed for a certain purpose other than the modelling of the geology itself. They are, for instance, widely used within sectors like environmental and groundwater administration, the hydrocarbon industry, mineral exploration and engineering geology (Berg et al. 2011). For groundwater administration purposes, the general 3D geological model has to be further developed into a hydrostratigraphical model that reflects hydraulic parameters rather than geology. This model then serves as an input for numerical modelling.

Three-dimensional geological models can be objectively constructed using geostatistical approaches or they can be subjectively constructed by knowledge-driven cognitive approaches. Including stochastic methods like multi-point geostatistical methods (Deutsch and Journel 1998; Strebelle 2002; Daly and Caers 2010), transition probability indicator simulation (Carle and Fogg 1996) or sequential indicator simulation (Deutsch and Journel 1998), the geostatistical approaches provide objective and documented models that may be constructed quickly, but they do not necessarily provide the most correct models in areas with few borehole data or with data of low quality (Wycisk et al. 2009). Also, for stochastic type modelling to perform at its maximum, it is necessary to describe all elements entering the models. However, this is very difficult or even impossible when dealing with elements in the category of "geological knowledge." Cognitive modelling (Royse 2010), on the other hand, ensures a high degree of incorporated geological background knowledge (Ross et al. 2005; Kessler et al. 2009; Wycisk et al. 2009; Royse 2010) such as the understanding of sedimentary processes, sequence stratigraphy or structural geology (Sharpe et al. 2002, 2003; Scharling et al. 2009). However, the inherited subjective nature of the interpretations in such models makes them difficult to document, and their credibility is difficult to evaluate by others. Furthermore, they are time consuming to establish. Therefore, a combined use of geostatistical and cognitive approaches may be the most suitable solution in many situations (Sharpe et al. 2007; Venteris 2007; Royse 2010; Stafleu et al. 2011; Raiber et al. 2012).

Three-dimensional geological models are often constructed as so-called layer-cake models or framework models. Such models are composed by a fixed number of continuous, stacked layers where the tops and bottoms are typically defined by the modelling geologist by regular grid surfaces interpolated from interpretation points and attached to layer boundaries in boreholes and/or on a series of cross-sectional profiles. This type of model is relatively simple to build in areas with continuous geology and where layered sequences dominate, but it has serious limitations in areas with complex geology (Turner 2006). The number of layers is limited due to difficulties in handling a high number of surfaces and their internal relationships, and modelling complex geology using this approach will therefore often result in loss of detail.

One way to meet the challenge of modelling complex geology is to make use of structured or unstructured 3D meshes (Turner 2006). Such meshes do not have the limitations of the layer-cake model. For example, in a voxel (volumetric pixel) model each voxel is assigned a lithology and/or another type of geological property. The most serious drawbacks of the voxel method are that, depending on the voxel size and discretisation method, it cannot describe perfect shapes and the precise location of, for example, layer boundaries and faults. Furthermore, it is difficult to construct such models manually and to incorporate cognitive interpretations. The benefits are that arbitrary shapes can be created depending on the voxel size and that statistical methods are easily applied to voxels because well-known 3D interpolation methods can be used to calculate properties for voxels by using information from, for example, boreholes or geophysics (Stafleu et al. 2011).

When AEM data are used in geological modelling, the primary source of information is linked to the electrical resistivity (inverse of conductivity). Converting resistivity data into geology is, however, not a straightforward task. Several, very different factors control the resistivity derived from AEM surveys, and a completely automated conversion routine has therefore not been established. In order to take these factors into account and make full use of the collected data, cognitive interpretation and modelling are preferable. In the present paper, we discuss this claim and we propose a method for cognitive interpretation and modelling. The method includes simple conversion of the data into pseudo-3D resistivity grids, and the use of a voxel modelling technique where models composed of regular as well as irregular voxel grids can be manually constructed while at the same time making full use of geological background knowledge.

\section{Airborne electromagnetic data}

\section{Data collection and processing}

The use of AEM for detailed geological mapping or other high-accuracy purposes is relatively new. The typical AEM high-accuracy application is used for groundwater exploration or other environmental purposes of which Siemon et al. (2009) gave a comprehensive overview. Various AEM systems are available for high-accuracy purposes, but most often either helicopter-borne frequency-domain systems or helicopter-borne time-domain systems are used. In the past decade, development has focused on the latter 
because they have superior depth penetration compared to the frequency-domain systems. Allard (2007) presented a thorough overview of the various helicopter-borne timedomain AEM systems, but the SkyTEM system (Sørensen and Auken 2004) represents an exception from other systems originally designed for mineral exploration, because the SkyTEM was purposely designed for mapping of geological structures in the near-surface for groundwater and environmental investigations.

Helicopter time-domain systems carry the transmitter loop (Tx) as a sling load beneath the helicopter. In the Tx loop, an electric current is abruptly terminated, causing a change of the primary magnetic field which, in turn, induces currents to flow in the ground. Because of ohmic loss, the currents decay and diffuse downward and outward in the subsurface. The change in decay rate of the secondary magnetic fields from these currents is picked up by an induction coil. In most cases, two perpendicular receiver coils $(\mathrm{Rx})$ pick up the inline field $\mathrm{x}$-component and the vertical field z-component.

For high-accuracy geological mapping, it is of crucial importance that all steps of the AEM data handling including processing and inversion are carried out to the highest standards. Some consequences of inaccurate processing or inversion are presented by Christiansen et al. (2011) and Viezzoli et al. (2013).

All AEM data to be used for high-accuracy geological mapping require post-processing before further use. The processing is often partly automatic and partly manual and related to two tasks. The first task is to process the altitude, inclination and position data in order to remove outliers and to provide continuity; especially, the altitude data need processing because in many cases they are affected by the vegetation on the surface (Beamish 2002; Auken et al. 2009). The second task is related to the distortion of data sets by the coupling responses from man-made installations. This is a very time-consuming process when operating in culturally developed areas and it takes up a significant part of the post-processing time (Auken et al. 2009). However, the removal of coupling-distorted data sets is crucial for the quality of the interpreted data sets.

When the data have been processed, they are generally inverted into a layered depth/resistivity model. Again, it is crucial that the inversion is carried out to the highest standards. Traditional smooth models (Constable et al. 1987) are a commonly used output. They most often utilise some sorts of lateral and vertical constraints to assure the expected coherency of the subsurface (Brodie and Sambridge 2006; Viezzoli et al. 2008) while stabilizing the inversion. For geological modelling, few-layered models producing sharp images of layer transitions are often a very valuable supplement (Jørgensen et al. 2005; Auken et al. 2008).
Airborne electromagnetic data can provide area-covering data sets with a high lateral density. Typically, a survey is flown with parallel lines with a distance of 100-500 m depending on the target and the complexity of the geology. The in-line sounding distance is typically $20-50 \mathrm{~m}$ as defined by the speed of the helicopter or aircraft combined with the stacking time to reach a certain signal/noise ratio. However, very often the data are resampled to offer much higher density at the cost of redundant data. Because data affected by man-made structures are removed in the postprocessing process, it is crucial to design the survey appropriately for the given survey area to obtain the needed amount of data. Jørgensen and Sandersen (2009) discussed aspects of data density considerations.

\section{$3 \mathrm{D}$ resistivity grids}

Although AEM data are typically inverted using both vertical and lateral constraints claiming to produce quasi2D or 3D models (Brodie and Sambridge 2006; Viezzoli et al. 2008), the results are discrete 1D models obtained along the flight lines, and these 1D models do not describe the resistivity properties between the flight lines. Brodie and Sambridge (2006) invert for the resistivity structures in a regular grid disconnected from the observation points, but are still relying on a 1D description. No 1D method will represent 3D structures correctly if lateral conductivity variations are very high (Goldman et al. 1994). However, along the flight lines, at least in the deeper parts, they can be considered as quasi-2D data because there will be a significant overlap between the footprints of neighbouring soundings. In many cases where the spacing between the flight lines is not too big, an overlap is also obtained between the soundings on neighbouring lines, thus providing a full 3D coverage in the deeper parts. In the more shallow parts where a limited overlap is present due to a smaller footprint, there will be no full $3 \mathrm{D}$ coverage. The lateral and vertical resolution is, on the other hand, much higher here.

In the process of geological modelling, it is typically very difficult to visualise and get an overview of the huge amount of resistivity data derived from AEM surveys. Each single 1D model and its corresponding processed data must be accessible for the modeller while modelling, but it is at least as important to somehow aggregate and generalise the data information in order to create the overview (Fig. 1). Since the AEM data do not provide a full 3D coverage of the entire subsurface, such generalisation must be performed by interpolation of the resistivity values from the 1D models. Different types of simple interpolated 3D resistivity grids can be made. Pryet et al. (2011) proposed a method in which the model parameters are first 2D interpolated and then converted into an irregular resistivity grid 

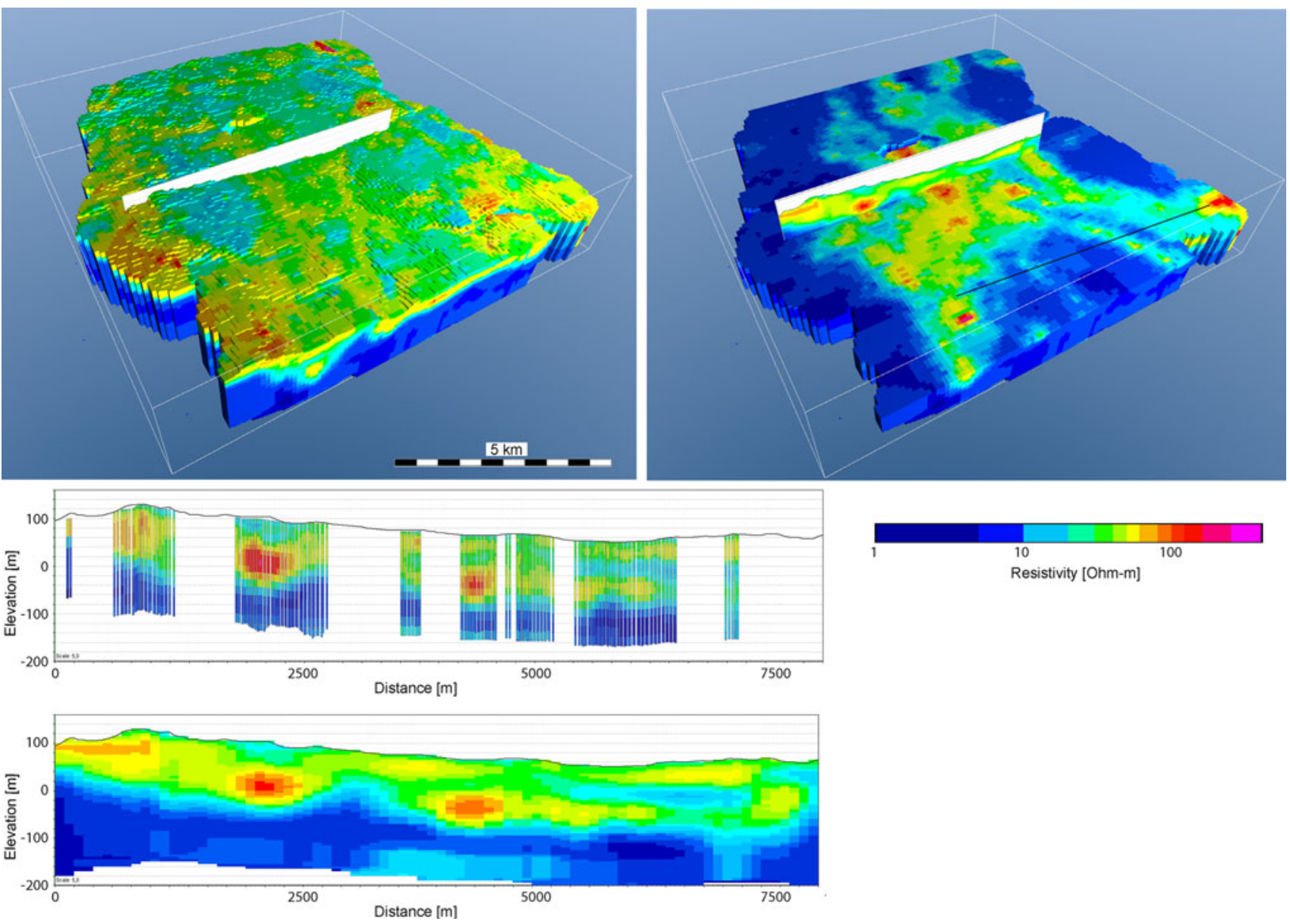

Fig. 1 Example of a 3D resistivity grid from airborne electromagnetic (SkyTEM) data (totally about 20,000 1D soundings covering an area of $120 \mathrm{~km}^{2}$ ). Upper left three-dimensional view of the resistivity grid unsliced. Upper right three-dimensional view of the resistivity grid sliced horizontally at $25 \mathrm{~m}$ below sea level. Middle single

by 3D gridding. Jørgensen et al. (2005) created 3D grids by stacking a series of horizontal 2D grids representing average resistivities derived from the 1D models at regular elevation intervals. Since our modelling concept requires regular 3D resistivity grids, we use the method described by (Jørgensen et al. 2005). The dimensions of the resistivity grid should aim at satisfying the maximum resolution of the data in order to incorporate as much detail as possible. Laterally, a quadratic cell size of 50 or $100 \mathrm{~m}$ is therefore an adequate choice, whereas the thickness of the cells should not exceed $5 \mathrm{~m}$. An example of a 3D resistivity grid is shown in Fig. 1. The grid is produced by first calculating weighted average resistivity values within elevation intervals of $5 \mathrm{~m}$ for each $1 \mathrm{D}$ model. These resistivity values are then interpolated from $\log$ (rho) values to a regular $2 \mathrm{D}$ grid by kriging, and these $2 \mathrm{D}$ grids are finally stacked into a 3D grid. The grid is prepared to cover the maximum interval of investigation by the AEM data. Because the grid is regular, it covers volumes well below electromagnetic soundings along a profile section, projected from a distance of $150 \mathrm{~m}$. Lower vertical slice through the resistivity grid. The cross section is shown in both $3 \mathrm{D}$ views above and is identical to the one shown in Fig. 2

the depth of investigation (DOI) in some areas. DOI is a calculated measure to be used within the process of geological interpretation and modelling (Christiansen and Auken 2012), and based on this, it is up to the modeller to judge to which depth the actual models are reliable for geological modelling.

The advantage of using a 3D grid is mainly that it allows the geologist to visualise and overview the data. The continuous representation of the data enhances the geologist's possibilities for image and pattern recognition. However, the grid does not always represent full 3D data coverage, therefore, it is always important to consider the search distance for the single 1D sounding data used for the interpolation of the grid. Sometimes a large spacing between individual soundings occurs, and the correlation across these gaps can be very weak.

The 3D grid can be sliced everywhere and visualised from all possible viewpoints (Fig. 1) and at the same time be compared with other data. 


\section{Translation from resistivity into lithology}

The electrical resistivity of sediments is mainly controlled by clay minerals and pore water ion content. According to Archie (1942), resistivity for clay-free sediments is inversely proportional to the pore water ion content, and if the pore water ion content then is known, indirect information about the content of clay minerals can be obtained. This information is dependent on the amount and type of clay (the cation-exchange capacity), and this relation constitutes the backbone in the process of geological interpretation. If the pore water ion content is constant throughout the different geological layers and structures, variations in clay content and type can be mapped. Furthermore, with a known specific ion content, estimates of these properties can be given. The typical measured resistivity values for common freshwater-saturated sediment types in Denmark are 2-12 ohm-m for marine Palaeogene clays, 10-40 ohm$\mathrm{m}$ for glaciolacustrine clays, $25-50 \mathrm{ohm}-\mathrm{m}$ for clay till, above $55 \mathrm{ohm}-\mathrm{m}$ for sand and gravel and above $80 \mathrm{ohm}-\mathrm{m}$ for limestone (Jørgensen et al. 2003). More detailed tables can be established locally for individual survey areas based on borehole data (Jørgensen et al. 2005; Sandersen et al. 2009).

To perform a geological interpretation of AEM data, a translation from geophysical model resistivity into lithology is needed. Without this translation, lithological properties cannot be assessed. The translation from resistivity into lithology can only be properly done if a series of important aspects regarding the physical properties of the subsurface and methodological limitations are carefully considered and implemented:

1. The degree of water saturation of the sediment

2. The ion content of the pore water

3. Clay content versus clay mineral type

4. Weak resolution of thin resistive layers and structures

5. Vertical resolution capability—thickness of layers versus depth

6. Horizontal resolution capability—size of structures versus depth

7. Depth Of Investigation (DOI)

8. Identification of coupled and otherwise noiseinfected soundings

9. Derived model uncertainty

10. The type of model used-few-layer or smooth model

11. Artefacts in $1 \mathrm{D}$ inversion due to $3 \mathrm{D}$ effects.

The degree of saturation (1) is important since without groundwater, the electrical conductivity derives only from clay minerals as there is no contribution from ions in the pore water. It is very important for the modeller to have information on the degree of saturation and the ion content of the pore water (2) and to include this information in the interpretations while modelling. Once these factors are known, low resistivities can be ascribed to clay, but it is generally impossible to discriminate between different clay types since the amount of clay/clay content is normally not known within a model layer (3). Clay can also be defined on the basis of the grain-size fraction $(<4 \mu \mathrm{m})$ and be composed of non-clay minerals. Such a type of sediment will show high resistivity even if it has many similarities with normal clay sediment types.

Since the nature of the electromagnetic data only enables limited resolution of high-resistive layers, resistivity values for sediments with resistivities above 100-200 ohm-m are not precisely determined (4). Discrimination between for instance silt/sand and gravel or limestone can therefore be difficult or impossible in some cases.

The resolution capability decreases with depth (5) for all electromagnetic diffusion methods. Our experience is that layers down to $2-5 \mathrm{~m}$ can optimally be resolved by AEM data in the shallow part, whereas at depths of around $100 \mathrm{~m}$ only layers thicker than $20-40 \mathrm{~m}$ are likely to be resolved. These numbers are strongly dependent on the resistivity of the layer sequence and the signal-to-noise ratio. Since the method cannot resolve thinner layers, individual geological layers will typically be merged into thicker model layers. Also based on our experience, no more than 4-6 layers can normally be resolved individually in a 1D sounding model, and the representation of a typical sedimentary sequence will therefore be a simplification of the truth, especially at deeper levels.

Also, the horizontal resolution capability (6) decreases with depth. At about $100 \mathrm{~m}$, the diameter of the footprint from which data are obtained exceeds $300-400 \mathrm{~m}$. At $25 \mathrm{~m}$, the footprint diameter is about 75-100 $\mathrm{m}$ (West and Macnae 1991). Therefore, small-scale spatial variations in geology are less well resolved at deeper levels than the shallower parts (Newman et al. 1986; Goldman et al. 1994).

The DOI (7) varies for different AEM systems, but it also varies within data sets obtained with the same system (Christiansen and Auken 2012). This variation arises because the DOI is strongly dependent on the resistivity of the subsurface, the flight height and the S/N ratio. Therefore, geological modelling to large depths without an estimate of DOI is complicated. The DOI calculation we use (Christiansen and Auken 2012) is data-driven, which allows the interpreter to distinguish between features originating primarily from data and features supported strongly by the lateral and/or vertical constraints. However, constraints are meant to impose continuity in areas with less data coverage by supplying information on the expected geological coherency. Therefore, it may still be very relevant to include information below the DOI into the geological model. 
It is also important that the modeller takes into account the issue of proper data processing (Viezzoli et al. 2013). Although the data set has been thoroughly processed, there will always be erroneous data left in the data set. Most of these data originate from high levels of background noise and coupling to man-made installations (8) (Sørensen et al. 2001). Of these, it can be difficult or impossible to identify all galvanic couplings during the data processing. Some of these couplings will often be left in the processed data, and the geophysical models will be distorted to some degree. Therefore, to avoid misinterpretation it is important to be aware of the possibility of coupled data during the modelling. Identification of coupled data is based on decay curve characteristics combined with maps of known electrical installations, roads, railroads, fences, houses, farms, etc. Some couplings are easily identified by unrealistic oscillations in the data, while other couplings are more tricky, and the removal of these will to some extent rely on the experience of the person performing the processing.

A model uncertainty estimate is normally provided by the inversion (9). During the modelling, this uncertainty estimate can be used to evaluate the credibility of the data and the inverted model, and to eventually exclude soundings or groups of soundings with high uncertainty and instead emphasise high-quality soundings. A further issue to be considered is the model types called few-layer models and smooth models (10). Both model types have some limitations, but each offers different advantages. The fewlayer model should mainly be used in the modelling when distinct layer boundaries have to be resolved or resistivity values are to be assessed for certain geological layers. The smooth model is better at describing details and spatial variations and will typically give a more realistic image of the geological setting. Both model types must be used in order to extract as much information from the data as possible. Certain 3D structures can produce artefacts in 1D inversions (11). These are often referred to as pant-legs effects that are shadows of the true structure dislocated from the true position (Goldman et al. 1994; Guillemoteau et al. 2012). The 3D effects are stronger when conductive structures overlie resistive structures than vice versa.

The challenges of converting electrical resistivity into lithology will be exemplified in the following. Figure 2 shows a cross section through a geologically complex glaciated area in the central part of Denmark. This area has been thoroughly mapped by mainly SkyTEM and deep drillings, and geological interpretations have been performed by combining with, and validation against, new and existing borehole data. The interpreted geology of the area was reported by Jørgensen et al. (2010). The upper cross section (Fig. 2a) shows a drawn sketch of the specific geological interpretation along the section (based mainly on the above-mentioned data). The profile illustrates the conceptual geology of the area, and it is this conceptual understanding of the geology that is aimed for when building the digital 3D geological model. From below, the geology on the profile is composed by limestone (green) overlain by marine Palaeogene clay (blue). This clay was heavily eroded by glaciers during the Pleistocene resulting in a complex glacigenic succession which to a large degree is composed of till (brown colours). Numerous incised buried valleys, glaciolacustrine clay units (orange), lenses of meltwater sand (red), and interglacial sand and clay (grey) occur within the till. The Pleistocene succession is subdivided into an upper and a lower unit by a regional unconformity (Fig. 2a).

A vertical slice of a $3 \mathrm{D}$ resistivity grid created from the SkyTEM data (smooth models) is shown on the next profile (Fig. 2b). The overall structure of the geological elements can be deduced from the coloured resistivity pattern on the profile. The marine Palaeogene clay, which is known to be very conductive, is clearly resolved by showing very low resistivities, whereas the glacial succession in general expresses higher resistivities. The sand-filled buried valleys, the sand lenses and the glaciolacustrine clay units within the glacial succession as seen in Fig. 2a occur as diffuse resistive and conductive structures. The unconformity occurs as a diffuse boundary throughout the profile, and the limestone is only partly resolved by a slight increase in resistivity in the lowermost part of the section.

To perform automated and direct conversion from resistivity into lithology, knowledge of the key intervals of resistivity values must be defined for the deposits in the area. These intervals cannot overlap in such a direct conversion, although they do that in reality. Based on the general experience with sediment resistivities for the region (Jørgensen et al. 2005), the sediments in our example could at best be classified in this way: $<8$ ohm-m $=$ Palaeogene clay; $8-25$ ohm-m = meltwater clay; $25-55$ ohm-m = clay till and $>55$ ohm-m $=$ meltwater sand and gravel.

The third profile (Fig. 2c) shows the result of the direct conversion into lithology according to this classification. From below, the limestone is not represented in the model at all since the resistivity overlaps the intervals of both Palaeogene clay and glaciolacustrine clay. Instead, in some places, the model indicates that the glaciolacustrine clay should be found below the Palaeogene. In the model, the otherwise sharp erosional contact between the Palaeogene clay and the glacial sediments is replaced by a $20-40-\mathrm{m}-$ thick layer of glaciolacustrine clay. This is caused by the difficulties of the smooth models to resolve this layer boundary exactly. Between 6,800 and 7,600 m, the Palaeogene clay is not very well resolved and, therefore, glaciolacustrine clay also occurs here. The deep borehole at $5,500 \mathrm{~m}$ indicates that the boundary is situated slightly deeper than indicated by the TEM. In general, the sand- 

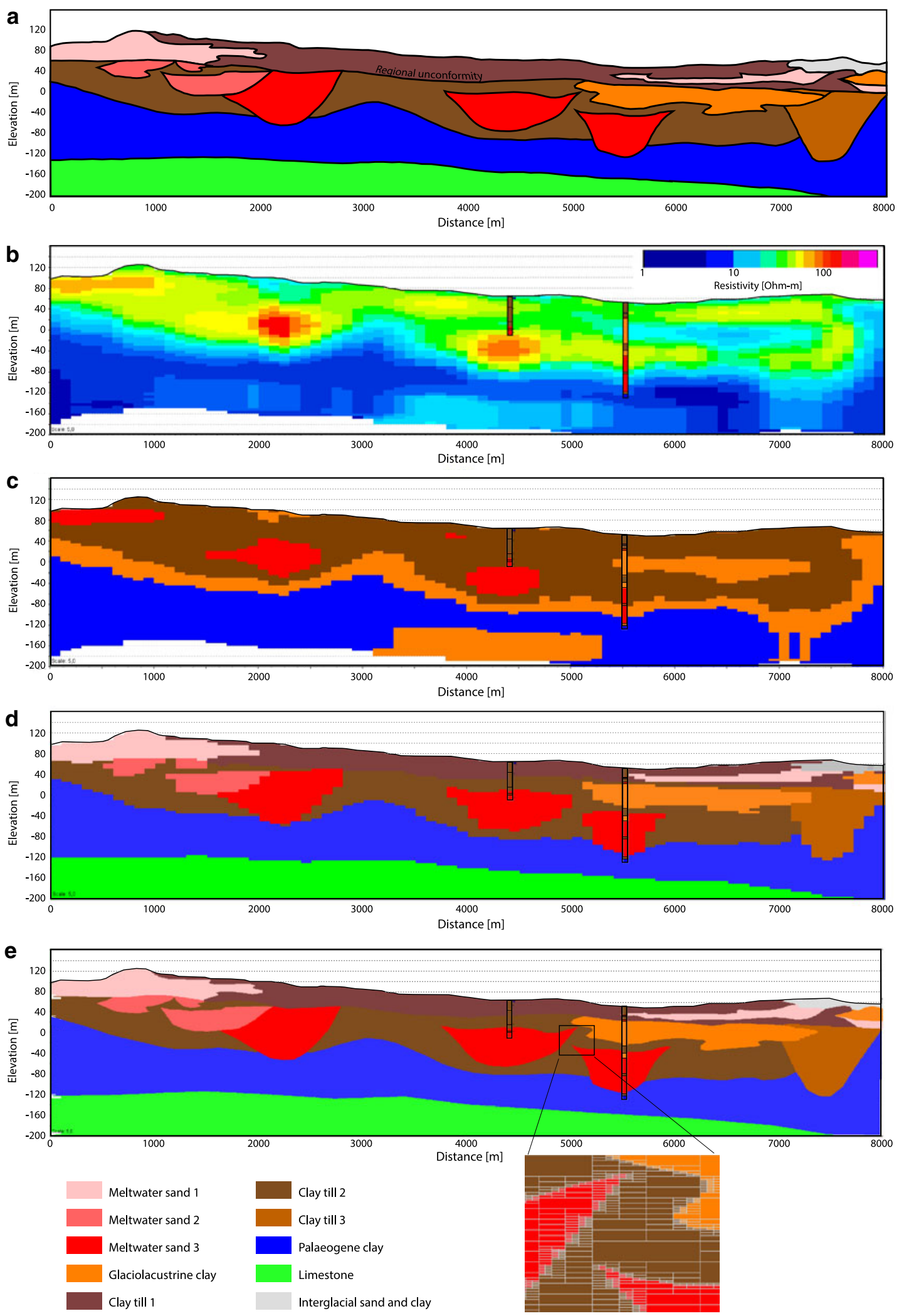

Fig. 2 Cross section through a 3D geological model showing differences in model types. a A sketch of the interpreted geology along the section, b resistivity data (3D grid), c lithology directly converted from resistivity, d cognitively constructed regular voxel model, e cognitively constructed octree voxel model with a close-up to show the octree mesh. Borehole data are shown in the cross sections 
filled buried valleys occur as oval-shaped features and not with distinct valley shapes. The clay-filled valley between 7,200 and 7,800 $\mathrm{m}$ does not occur in the model because the infill does not differ in resistivity from the surroundings. The same applies for the interglacial unit above this valley. Also, the buried valley between 5,000 and 6,000 $\mathrm{m}$ does not occur in the model. This valley is insufficiently resolved and does not appear with resistivities that contrast with those of the surrounding sediments. The regional unconformity is also not seen, and the sand lens close to the surface between 5,400 and $7,600 \mathrm{~m}$, which is too thin to be satisfactorily resolved in the resistivity grid, does not occur either.

As seen from the example above, the model created by the automated conversion does not satisfactorily represent and outline the expected geology along the profile. The model can be improved by incorporating geological background knowledge and by carefully considering the abovementioned 11 aspects during cognitive modelling.

\section{Modelling concept}

In order to be able to capture the modeller's cognitive interpretations during model construction, we have developed a dedicated voxel modelling concept. This concept makes use of the geophysical information as defined by the $3 \mathrm{D}$ resistivity grid, but the conversion into lithology is done manually step by step and not by automated routines. However, when selecting voxels for manual editing, the sheer number of voxels to be manipulated raises problems as editing every single one is very time consuming. The dedicated tools, which have been developed as a part of the Geoscene3D 2013 software package (Geoscene3D http:// www.geoscene3d.com), are designed to handle voxels in a more flexible way. The modelling concept and the developed tools are described in the following.

Basically, the $3 \mathrm{D}$ resistivity grid can be regarded as a voxel grid with a resistivity attribute. Voxel grids can hold an unlimited number of attributes and different parameters can be added to the grid structure as attributes. One attribute could be lithology. Other attributes could be facies or model uncertainty. The challenge for the modeller is to select a volume in the 3D space on the basis of resistivity values combined with geological background knowledge and information from other data, and then to assign a chosen lithology and/or other attributes to the selected volume. After volume and attribute selection, the volume will be filled with the interpreted value for each attribute.

The selection of volumes can be done in the following ways:

- By a horizontal polygon digitized in a map window. The volume can be limited vertically by a top and bottom level defined by the user or by surfaces. One or more polygons can be used for the horizontal selection. Each region can either include or exclude the area inside the polygon.

- By a vertical polygon digitized in a vertical cross section window. The volume can be limited laterally by a selected buffer distance orthogonal to the cross section plane.

- Surfaces can limit the volume vertically by using their $Z$ node values, or horizontally by using their areal coverage.

- By "region grow" techniques in regular 3D grids (Fig. 3). The region grow function selects all voxels that are connected to a chosen "seed" voxel and within a given interval. When using resistivity grids and a resistivity interval of, for example, 15-30 ohm-m, the selected volume will grow from the seed voxel until it reaches a limit as defined by voxels with resistivities within the interval.

- The volume can be limited using $\mathrm{X}, \mathrm{Y}$ or $\mathrm{Z}$ coordinates as either minimum or maximum.

- If the volume shape is known and the shape is available in a digital compatible format, the known shape can be imported and used for selection.

- By single voxel selection with mouse tools.

All the selection methods described above can be combined freely. As an example, a region grow selection can be further limited horizontally by one or more polygons and vertically by a top and bottom surface. The techniques all fill empty voxels. However, existing voxels can be selected for modification or deletion in a number of ways.

The manual voxel editing can be operated and visualised synchronously in map sections, vertical cross sections and directly in a $3 \mathrm{D}$ space.

Figure $2 \mathrm{~d}$ shows an example of a cognitively constructed regular voxel model where the tools described above have been used. From a comparison with the sketch of the expected geology in Fig. 2a, it can be seen that the expected geology has been well reproduced, although the blocky appearance of the voxels hampers the delineation of distinct boundaries between the lithological units. The geology is much better reproduced here in this voxel model than was the case in the directly converted model (Fig. 2c). The limestone and the Palaeogene clay are modelled by using manually constructed surfaces as limits. The other units above the clay are selected and modelled one by one using different combinations of polygon and region limits, region grow, single voxel selection, borehole data constraints and surface limitation.

The main weakness of the regular voxel model as shown in Fig. $2 \mathrm{~d}$ is its limited ability to describe exact and smooth layer boundaries and details smaller than the chosen voxel 

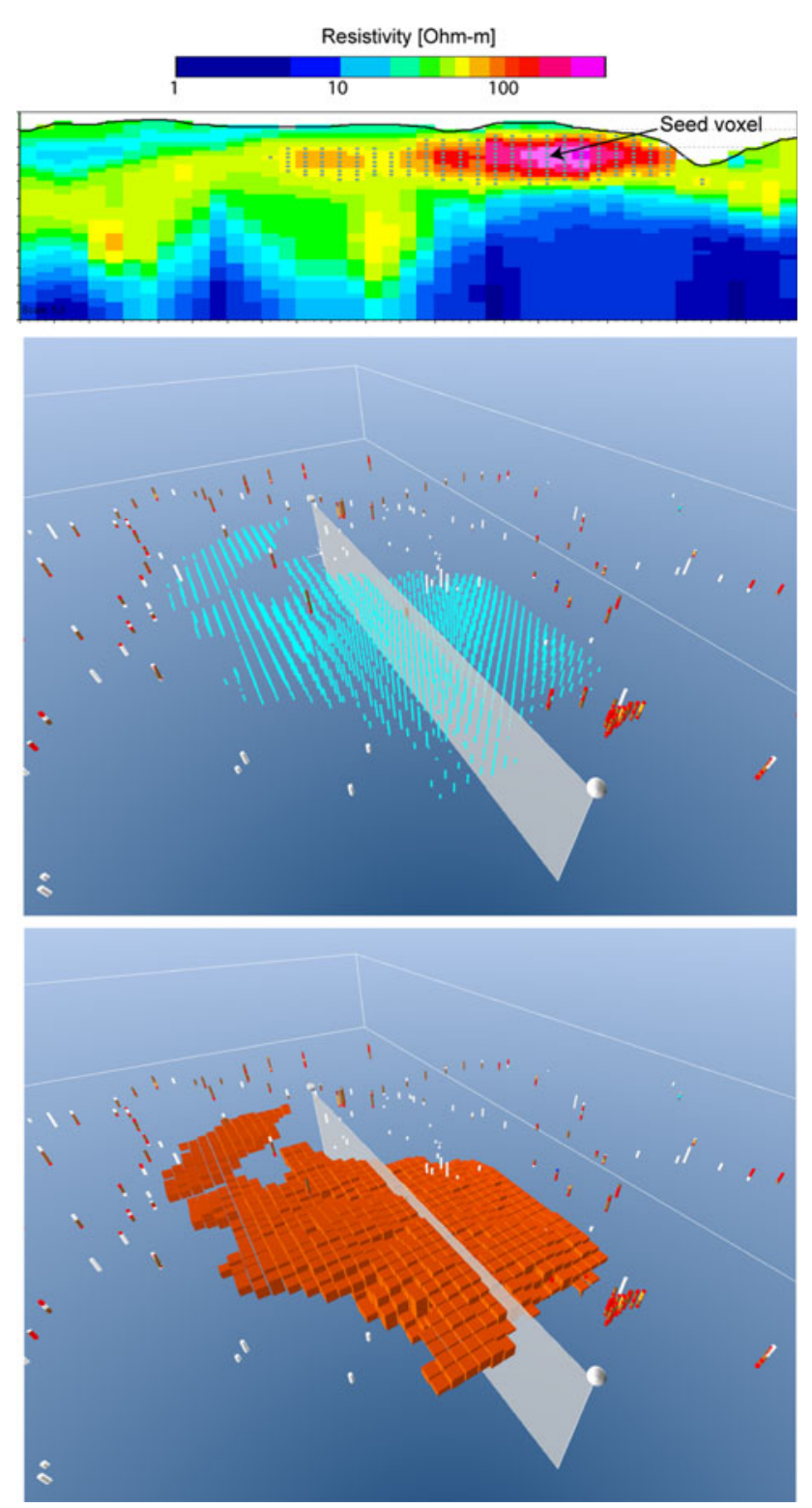

Fig. 3 Selection of voxel nodes by using the region grow tool. Above the 3D resistivity grid sliced vertically along a profile section. A seed voxel is chosen on this vertical section, and a group of neighbouring voxels within a certain resistivity interval is selected (blue dots). Middle three-dimensional view of all selected voxel nodes (blue dots) and borehole data. Below three-dimensional view after assigning the selected voxels a property (e.g., meltwater sand)

size. The boxy square look of a voxel does not always please a modeller, who likes to think in layers and more nicely shaped geological features.

\section{Octree modelling}

The level of detail and the detail in geological knowledge are usually highest near the terrain surface and lowest in the deeper parts of a model. The level of detail often varies
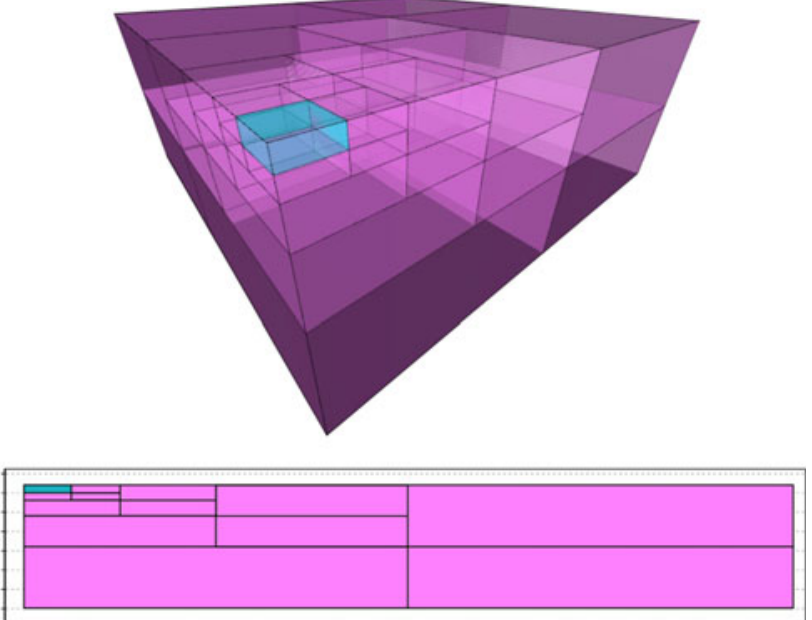

Fig. 4 An octree-based voxel model from GeoScene 3D. The upper image shows the 3D object with a fine voxel resolution in the left top corner. Below: a cross section through the same object showing the fining in resolution towards the upper left

across the model, as does the geological variation. The resolution of the grid must be chosen from the start and is often a compromise between the smallest size of the features to be modelled, the data resolution and the size of the model area. The voxel resolution and the size of the area are important issues because the amount of voxels (typically millions) will put stress on both hardware and software if such an amount is to be handled in a real-time 3D environment. To minimise the problems with resolution and performance, the GeoScene 3D software has been developed to handle irregular voxels by using the "octree" algorithm, a tree data structure in which each internal node has exactly eight children (Meagher 1980). In this approach, all voxels can be divided down as many times as needed. The octree algorithm takes care of a mechanism that subdivides the voxel into eight equally sized smaller voxels (Fig. 4). These new voxels can then be further subdivided in the same way.

So, depending on which level of detail is desired, a very fine resolution can be chosen for certain areas in the model volume and a very coarse resolution can be chosen in other areas. In theory, voxels at $\mathrm{mm}$ scale can be combined with voxels at $\mathrm{km}$ scale. The octree model can therefore reflect huge variations in geology and knowledge across the same model, while at the same time maintaining a limited number of voxels.

Another benefit of the octree approach is that this algorithm can be used to quickly display large data sets in a 3D visualisation by visualising large voxels (the top level in the octree) for objects in the background and more detailed voxels for objects that are closer to the viewer.

Modelling of the octree voxel model is somewhat similar to editing the regular voxels model as described above. 
However, the octree approach allows some additional tools to be used. One of these tools is designed to subdivide a voxel or groups of voxels into smaller voxels in areas where the modeller needs more detail.

When the octree concept is applied to the model shown in Fig. 2, much smoother boundaries between the units are achieved (Fig. 2e). The features resemble the expected geology (Fig. 2a) more convincingly and with a possibility of having more detail than the regular voxel model (Fig. 2d).

Instead of improving the resolution of the model along layer boundaries and detailed areas, another option is just to keep the resolution as it is in the regular model, although converted into an octree model. By converting the regular voxel model shown in Fig. 2 into an octree model, the number of voxels can be reduced by $84 \%$, simply because the voxel size is dramatically extended in areas with homogeneous geology.

\section{Discussion}

Advances in AEM technology provide new opportunities for collecting high-quality, spatially dense subsurface data. These data enable mapping and interpretation of a hitherto unseen amount of geological detail in heterogeneous settings such as glaciated areas. This new geological knowledge gives geologists the opportunity to build very detailed 3D geological models. Detailed models based on AEM data can be constructed by automated geostatistical approaches (Bosch et al. 2009; Gunnink et al. 2012), but such approaches cannot, as described above, fully account for all the essential physical limitations of the AEM method as well as all geological background knowledge. These limitations and potentials are tackled and included within the presented approach of iterative, manual geological voxel model building based on 3D resistivity grids, but the approach requires highly performing, sophisticated and dedicated selection and visualisation tools. The benefits of using the developed tools spring from the fact that it is possible to build highly detailed models while at the same time taking the limitations of the AEM data into account and using the potential to incorporate geological background knowledge.

The presented approach is more time consuming than an approach that relies on automated, statistics-based methods. Another limitation is that even if the modelling is performed very carefully, it will still be impossible to incorporate all information from AEM data into the models. Therefore, some details will be left in the data set after the modelling process. Automated, statistic-based methods can more easily make use of all data information and produce highly detailed models, but since they cannot fully account for the limitations and geological background knowledge, the models produced will not necessarily come up with the best solutions everywhere. A third limitation of the presented modelling approach lies in the problem of documentation, because cognitive data interpretation involves a high degree of subjectivity that is difficult to document and evaluate with respect to uncertainty. Uncertainty evaluation must be done by using subjective approaches as described in, for example, Sandersen (2008).

Geostatistical methods are able to provide other sorts of information that cannot be derived during the manual modelling approach as presented here. Certain data trends and distributions may, for instance, occur during the geostatic processing of the data, and the presented cognitive approach may therefore successfully be supplemented by geostatistical methods. Simple geostatistical processing of the AEM has already been performed by the interpolation of the $3 \mathrm{D}$ resistivity grid. However, further supplementation with geostatistical methods may afford the geologist with a possibility for improving the efficiency of the modelling and the documentation, but possibly also its precision and quality. Combined cognitive and geostatistical modelling have proven successful by, for example, Venteris (2007) and Bajc and Newton (2007).

The need for including details in 3D models places high demands on the software and hardware used, and it is essential that software and hardware performance meet the chosen level of detail. Visualisation, navigation and editing must be possible to execute without too much response time as it is essential to keep the manual and iterative modelling procedure running effectively and uninterrupted. The performance is also stressed in this type of model building because the modeller is continuously requested to interact with the computer through visualisation, selection, execution/saving and navigation. One way to increase the level of detail without losing performance is to construct the octree voxel models instead of regularly discretised voxel models. Preliminary tools for manual model construction of octree models have been developed with promising results as shown here. Therefore, further development of the manual voxel modelling approach will be based on the octree algorithm. Other developments may include improvement of the facilities for optimised 3D visualisation of resistivity data together with other data as well as the voxel model grid itself as these elements form the basis for the modeller's cognitive interpretation. Future research should also continue the development of tools with which an exact, desired group of voxels can be effectively and precisely selected and converted into geology.

\section{Conclusions}

Airborne electromagnetic data provide detailed geological information for 3D geological model construction, but in 
order to optimise the amount and quality of information gained from the data at least 11 different types of physical limitations of the AEM method must be acknowledged and considered during data interpretation. In addition to this, geological background knowledge has to be utilised during the modelling.

To include this and to gain as much as possible information from the TEM data, we have presented a cognitive approach that includes manual modelling. With this approach, we have suggested that the model should be constructed iteratively as a voxel model by utilising the spatial nature of the collected resistivity data through the construction of 3D resistivity grids. Dedicated software tools to support the modelling approach have been developed as part of the GeoScene 3D software package. Octree voxel modelling has been successfully tested and integrated in the approach in order to increase model precision and performance.

Acknowledgments The software development is a part of the HYACINTS research project funded by The Danish Council for Strategic Research, Danish Agency for Science Technology and Innovation. Jens Christian Refsgaard is thanked for his helpful comments on an early version of the paper. Two anonymous reviewers are thanked for their reviews and helpful comments.

\section{References}

Allard M (2007) On the Origin of the HTEM Species. In: Milkereit B (ed) Proceedings of exploration 07: fifth decennial international conference on mineral exploration, pp 355-374

Archie GE (1942) The electrical resistivity $\log$ as an aid in determining some reservoir characteristics. Trans AIME 146: $54-62$

Auken E, Christiansen AV, Jacobsen L, Sørensen KI (2008) A resolution study of buried valleys using laterally constrained inversion of TEM data. J Appl Geophys 65(1):10-20

Auken E, Christiansen AV, Westergaard JA, Kirkegaard C, Foged N, Viezzoli A (2009) An integrated processing scheme for highresolution airborne electromagnetic surveys, the SkyTEM system. Explor Geophys 40:184-192

Bajc AF, Newton MJ (2007) Mapping the subsurface of waterloo region, Ontario, Canada; an improved framework of quaternary geology for hydrogeological applications. J Maps 2007:219-230. doi:10.4113/jom.2007.56

Beamish D (2002) The canopy effect in airborne EM. Geophysics 67(6):1720-1728. doi:10.1190/1.1527073

Berg CB, Mathers SJ, Kessler H, Keefer DA (2011) Synopsis of Current Three-dimensional Geological Mapping and Modeling in Geological Survey Organizations, vol 578. Circular, Illinois State Geological Survey, Illinois

Bosch JHA, Bakker MAJ, Gunnink JL, Paap BF (2009) Airborne electromagnetic measurements as basis for a 3D geological model of an Elsterian incision. Zeitschrift der Deutschen Gesellschaft für Geowissenschaften 160(3):249-258. doi:10. 1127/1860-1804/2009/0160-0258

Brodie R, Sambridge M (2006) A holistic approach to inversion of frequency-domain airborne EM data. Geophysics 71(6):G301G312
Carle SF, Fogg GE (1996) Transition probability-based indicator geostatistics. Math Geol 28(4):453-476. doi:10.1007/bf02083656

Christiansen AV, Auken E (2012) A global measure for depth of investigation. Geophysics 77(4):WB171-WB177

Christiansen AV, Auken E, Viezzoli A (2011) Quantification of modeling errors in airborne TEM caused by inaccurate system description. Geophysics 76(1):F43-F52. doi:10.1190/1.3511354

Constable SC, Parker RL, Constable CG (1987) Occam's inversion: a practical algorithm for generating smooth models from electromagnetic sounding data. Geophysics 52(3):289-300

Daly C, Caers J (2010) Multi-point geostatistics - an introductory overview. First Break 28(9):39-47. doi:10.3997/1365-2397. 2010020

Deutsch CV, Journel AG (1998) GSLIB: geostatistical software library and user's guide, 2nd edn. Oxford University Press, Oxford

Geoscene3D (2013) (http://www.geoscene3d.com) November 6th http://www.geoscene3d.com. Accessed April 15th 2013

Goldman M, Tabarovsky L, Rabinovich M (1994) On the influence of 3-D structures in the interpretation of transient electromagnetic sounding data. Geophysics 59(6):889-901

Guillemoteau J, Sailhac P, Behaegel M (2012) Fast approximate 2D inversion of airborne TEM data: Born approximation and empirical approach. Geophysics 77(4):89-97. doi:10.1190/ geo2011-0372.1

Gunnink JL, Bosch JHA, Siemon B, Roth B, Auken E (2012) Combining ground-based and airborne EM through artificial neural networks for modelling glacial till under saline groundwater conditions. Hydrol Earth Syst Sci 16:3061-3074

Jørgensen F, Sandersen PBE (2009) Buried valley mapping in Denmark: evaluating mapping method constraints and the importance of data density. Zeitschrift der Deutschen Gesellschaft für Geowissenschaften 160(3):211-223. doi:10.1127/ 1860-1804/2009/0160-0211

Jørgensen F, Sandersen P, Auken E (2003) Imaging buried quaternary valleys using the transient electromagnetic method. J Appl Geophys 53(4):199-213

Jørgensen F, Sandersen PBE, Auken E, Lykke-Andersen H, Sørensen $\mathrm{K}$ (2005) Contributions to the geological mapping of Mors, Denmark-A study based on a large-scale TEM survey. Bull Geol Soc Den 52:53-75

Jørgensen F, Møller RR, Sandersen PBE, Nebel L (2010) 3-D geological modelling of the Egebjerg area, Denmark, based on hydrogeophysical data. Geol Surv Den Greenl Bull 20:27-30

Jørgensen F, Scheer W, Thomsen S, Sonnenborg TO, Hinsby K, Wiederhold H, Schamper C, Burschil T, Roth B, Kirsch R, Auken E (2012) Transboundary geophysical mapping of geological elements and salinity distribution critical for the assessment of future sea water intrusion in response to sea level rise. Hydrol Earth Syst Sci 16(7):1845-1862. doi:10.5194/hess-161845-2012

Kessler H, Mathers S, Sobisch HG (2009) The capture and dissemination of integrated 3D geospatial knowledge at the British Geological Survey using GSI3D software and methodology. Comput Geosci 35(6):1311-1321. doi:10.1016/j.cageo. 2008.04.005

Meagher D (1980) Octree encoding: a new technique for the representation, manipulation and display of arbitrary 3-D objects by computer. Technical report IPL-TR-80-111. Renssel Polytechnic Institute

Newman GA, Hohmann GW, Anderson WL (1986) Transient electromagnetic response of a three-dimensional body in a layered earth. Geophysics 51(8):1608-1627

Oldenborger GA, Pugin A, Pullan SE (2013) Airborne time-domain electromagnetics, electrical resistivity and seismic reflection for regional three-dimensional mapping and characterization of the 
Spiritwood Valley Aquifer, Manitoba Canada. Near Surf Geophys 11(1):63-74. doi:10.3997/1873-0604.2012023

Pryet A, Ramm J, Chiles JP, Auken E, Deffontaines B, Violette S (2011) 3D resistivity gridding of large AEM datasets: a step toward enhanced geological interpretation. J Appl Geophys 75:277-283. doi:10.1016/j.jappgeo.2011.07.006

Raiber M, White PA, Daughney CJ, Tschritter C, Davidson P, Bainbridge SE (2012) Three-dimensional geological modelling and multivariate statistical analysis of water chemistry data to analyse and visualise aquifer structure and groundwater composition in the Wairau Plain, Marlborough District, New Zealand. J Hydrol 436:13-34. doi:10.1016/j.jhydrol.2012.01.045

Ross M, Parent M, Lefebvre R (2005) 3D geologic framework models for regional hydrogeology and land-use management: a case study from a Quaternary basin of southwestern Quebec Canada. Hydrogeol J 13(5-6):690-707. doi:10.1007/s10040-004-0365-x

Royse KR (2010) Combining numerical and cognitive 3D modelling approaches in order to determine the structure of the Chalk in the London Basin. Comput Geosci 36(4):500-511. doi:10.1016/j. cageo.2009.10.001

Sandersen PBE (2008) Uncertainty assessment of geological models a qualitative approach. In: Refsgaard JC, Kovar K, Haarder E, Nygaard E (eds) 2008. Credibility of Modelling. IAHS Publication, Calibration and Reliability in Groundwater modelling, pp 337-344

Sandersen PBE, Jørgensen F, Larsen NK, Westergaard JH, Auken E (2009) Rapid tunnel-valley formation beneath the receding Late Weichselian ice sheet in Vendsyssel Denmark. Boreas 38(4): 834-851. doi:10.1111/j.1502-3885.2009.00105.x

Scharling PB, Rasmussen ES, Sonnenborg TO, Engesgaard P, Hinsby K (2009) Three-dimensional regional-scale hydrostratigraphic modeling based on sequence stratigraphic methods: a case study of the Miocene succession in Denmark. Hydrogeol $\mathrm{J}$ 17(8):1913-1933. doi:10.1007/s10040-009-0475-6

Sharpe DR, Hinton MJ, Russell HAJ, Desbarats AJ (2002) The need for basin analysis in regional hydrogeological studies: oak Ridges Moraine Southern Ontario. Geosci Can 29(1):3-20

Sharpe DR, Pugin A, Pullan SE, Gorrell G (2003) Application of seismic stratigraphy and sedimentology to regional hydrogeological investigations: an example from Oak Ridges Moraine, southern Ontario Canada. Can Geotech J 40(4):711-730. doi:10. $1139 / \mathrm{t} 03-020$

Sharpe D, Russell HAJ, Logan C (2007) A regional 3-dimensional geological model of the Oak Ridges Moraine area, Ontario, Canada. J Maps 2007:239-253. doi:10.4113/jom.2007.58
Siemon B, Christiansen AV, Auken E (2009) A review of helicopterborne electromagnetic methods for groundwater exploration. Near Surf Geophys 7(5-6):629-646

Sørensen KI, Auken E (2004) SkyTEM-A new high-resolution helicopter transient electromagnetic system. Explor Geophys 35:191-199

Sørensen KI, Effers $\emptyset$ F, Auken E (2001) A hydrogeophysical Investigation of the Island of Drejø. Europ J Environ Eng Geophys 6:109-124

Stafleu J, Maljers D, Gunnink JL, Menkovic A, Busschers FS (2011) $3 \mathrm{D}$ modelling of the shallow subsurface of Zeeland, the Netherlands. Neth J Geosci-Geologie En Mijnbouw 90(4): 293-310

Strebelle S (2002) Conditional simulation of complex geological structures using multiple-point statistics. Math Geol 34(1):1-21. doi:10.1023/a:1014009426274

Troldborg L, Refsgaard J, Jensen K, Engesgaard P (2007) The importance of alternative conceptual models for simulation of concentrations in multi-aquifer system. Hydrogeol J 15:843-860

Troldborg L, Jensen K, Engesgaard P, Refsgaard J, Hinsby K (2008) Using environmental tracers in modeling flow in a complex shallow aquifer system. J Hydrol Eng 12(11):1037-1048

Turner AK (2006) Challenges and trends for geological modelling and visualisation. Bull Eng Geol Environ 65(2):109-127. doi:10. 1007/s10064-005-0015-0

Venteris ER (2007) Three-dimensional modeling of glacial sediments using public water-well data records: an integration of interpretive and geostatistical approaches. Geosphere 3(6):456-468. doi: $10.1130 /$ ges00090.1

Viezzoli A, Christiansen AV, Auken E, Sørensen KI (2008) Quasi-3D modeling of airborne TEM data by spatially constrained inversion. Geophysics 73(3):F105-F113

Viezzoli A, Jørgensen F, Sørensen C (2013) Flawed processing of airborne EM data affecting hydrogeological interpretation. Ground Water. doi:10.1111/j.1745-6584.2012.00958.x

West GF, Macnae JC (1991) Physics of the electromagnetic induction exploration method. In: Nabighian MN, Corbett JD (eds) Electromagnetic methods in applied geophysics, vol 2. Investigations in geophysics, Society of exploration geophysicists, pp 5-45

Wycisk P, Hubert T, Gossel W, Neumann C (2009) High-resolution 3D spatial modelling of complex geological structures for an environmental risk assessment of abundant mining and industrial megasites. Comput Geosci 35(1):165-182. doi:10.1016/j.cageo. 2007.09.001 